\title{
Subject Index
}

Antisecretory factor, milk 17

Bifidobacterium infantis, breast milk

tryptophan interactions 107-109

Breastfeeding

benefits

maternal 22

neonatal 22, 23

immunological connection between mother and infant 39

neonatal risks 23,24

noncommunicable disease protection in later life 66, 67

Casein, evolution 6, 7

CD14, milk 17, 18, 99

Colostrum, see Immunology, milk, and lactation

Evolution, see Lactation evolution

Exosomes, milk 100

FDSCP, see Follicular dendritic cell secreted peptide

Follicular dendritic cell secreted peptide (FDSCP) 6

Fucosyltransferase (FUT)

functional overview 125

genotypes and neonatal immunity 62 , 63

variants and milk oligosaccharide composition 118, 119

FUT, see Fucosyltransferase
Gut microbiome, see Microbiome

House dust mite, allergic sensitization through breast milk 54, 55

Immunoglobulins, milk

activity 16, 17, 42, 43

antigens 30

autoantibodies 43

induction by immunization 31, 32

milk microbiota effects 99

mucosal immunity 32-34, 61

reactivity 17

secretory IgA

advantages in human milk 29

glycan-mediated binding 160-163

immune exclusion 159, 160

infant commensal colonization role $164-166,171$

infection prevention 163, 164

production 159

specificity and antigenic stimulation

30,31

types $16,28,29$

Immunology, milk, and lactation

chemokines 18

colostrum 16

cytokines 18, 39-41, 62

epithelial cells 19, 43

growth factors 18,100

historical perspective 11-15, 60, 61

Immunoglobulins, see

Immunoglobulins, milk

infection prevention 51, 52

leukocytes 18 
long-term susceptibility to immune-

dependent diseases 52-55

macrophages 19

mucosal immunity 32-34

osteopontin in immune development 155,156

prospects for study $34-36$

stem cells $19,100,101$

T cells 19, 20, 44

Lactation evolution

casein evolution 6,7

mammary gland evolution 3-5

milk fat globule evolution 7, 8

milk oligosaccharide evolution 8,9

overview 2-4

Lactoferrin

gut microbe interactions 143-145

milk composition and function 141-143

preterm infant studies

ELFIN trial 147-149

late-onset sepsis prevention 145 ,

146-149

necrotizing enterocolitis

prevention 145, 147-149

Late-onset sepsis (LOS)

lactoferrin prevention 145, 146-149

overview 142, 143

LOS, see Late-onset sepsis

Malaria, breast milk antigens 55

Mammary gland evolution 3-5

Mammopilosebaceous unit (MPSU), formation 5, 59, 60

Metabolome milk fat globule membrane concentrates 137,138

MFG, see Milk fat globule

Microbiome

human milk

clinical significance 72,73

immunoglobulin effects 99

metabolomics 97, 98

necrotizing enterocolitis

prevention 104, 106-110

origins $77-81$

overview $68,69,76,77,96,97$ infants

gut colonization with breast

feeding $67-70$

lactoferrin interactions 143-145

milk fat globule membrane concentrate 137,138

milk secretory IgA and colonization 164-166, 171

mastitis prevention in nursing 81-84

Milk fat globule (MFG)

evolution 7,8

membrane

concentrate supplementation effects in infants 137, 138

infections in infants and children 134-137

prospects 138,170

structure and synthesis 134

overview 133, 134

Milk microbiome, see Microbiome

Milk oligosaccharides, see

Oligosaccharides, milk

Milk virome, see Virome, milk

MPSU, see Mammopilosebaceous unit

NEC, see Necrotizing enterocolitis

Necrotizing enterocolitis (NEC)

immune responses 105

lactoferrin prevention 145, 147-149

milk microbiota in prevention 104 ,

106-110

milk secretory IgA prevention 165

overview 104, 142

short-chain fatty acid studies 110

Norovirus

oligosaccharide binding inhibition

dendrimer studies 129, 130

fucoidan binding 130, 131

in-solution binding experiments

128, 129

neoglycolipid array identification

of norovirus binders 126, 127

overview 125,126

ODAM, see Odontogenic, ameloblast-

associated protein

Odontogenic, ameloblast-associated

protein (ODAM) 6 
Oligosaccharides, milk

composition 116, 117

digestion 119

evolution 8,9

functions 119-121

norovirus binding inhibition

dendrimer studies 129, 130

fucoidan binding 130, 131

in-solution binding experiments

128, 129

neoglycolipid array identification

of norovirus binders 126, 127

overview 125, 126

synthesis, see Fucosyltransferase

therapeutic applications 121, 122

Oligosaccharides, see Milk

oligosaccharides

OPN, see Osteopontin

Osteopontin (OPN)

milk

functions 153, 170, 171

immune development role 155

intestinal proliferation and maturation role 153,154

neurodevelopment promotion 154, 155

overview 152, 153

OVA, see Ovalbumin

Ovalbumin (OVA), allergy prevention in mice through breastfeeding 53, 54
Preterm infants, see Late-onset sepsis; Necrotizing enterocolitis

Rotavirus, milk studies 22, 120, 135

SCFAs, see Short-chain fatty acids

SCPPs, see Secretory calcium-binding phosphoproteins

Secretory calcium-binding phosphoproteins (SCPPs) 6, 60

Short-chain fatty acids (SCFAs), necrotizing enterocolitis studies 110

T cells

milk 19, 20, 44

Th2 responses and allergy 49, 50

TLRs, see Toll-like receptors

Toll-like receptors (TLRs), milk 17, 18, 99

Virome, milk

diversity and taxonomy 87,88

prospects for study 91,92

role in infant health $89-91$

transmission 88,89

Xanthine oxidoreductase (XOR) 7, 8

$\mathrm{XOR}$, see Xanthine oxidoreductase 